\title{
Baixa riqueza zooplanctônica indicando condições adversas de seca e eutrofização em um reservatório no Nordeste do Brasil
}

\author{
Caio O. Silva' (D), Adjuto R. Junior ${ }^{2}$ (D), Gilmar Perbiche-Neves ${ }^{3}$ (D), \\ Allysson P. Pinheiro' (1) \& Sírleis R. Lacerda ${ }^{4}$ (1)
}

\begin{abstract}
1. Laboratório de Crustáceos do Semiárido, Departamento de Ciências Biológicas, Centro de Ciências Biológicas e da Saúde, Universidade Regional do Cariri, Rua Cel. Antônio Luiz, 1161, Pimenta, 63105-000 Crato, CE, Brasil. (caioolivirasilva@gmail.com)

2. Laboratório de Ecologia e Microbiologia de Ecossistemas Aquáticos, Departamento de Microbiologia e Parasitologia, Centro de Biociências, Universidade Federal do Rio Grande do Norte, Campus Universitário, BR-101, Lagoa Nova, 59072-000 Natal, RN, Brasil. (jr_arangel@hotmail.com)

3. Laboratório de Plâncton, Departamento de Hidrobiologia, Centro de Ciências Biológicas e da Saúde, Universidade Federal de São Carlos, Jardim Guanabara,

13565-905 São Carlos, SP, Brasil. (gilmarperbiche83@gmail.com)

4. Laboratório de Botânica, Departamento de Ciências Biológicas, Centro de Ciências Biológicas e da Saúde, Universidade Regional do Cariri, Rua Cel. Antônio Luiz, 1161, Pimenta, 63105-000 Crato, CE, Brasil. (sirleisrl@gmail.com)
\end{abstract}

Recebido 31 agosto 2019

Aceito 9 abril 2020

Publicado 05 junho 2020

DOI 10.1590/1678-4766e2020009

\begin{abstract}
Low zooplankton richness indicating adverse drought and eutrophication conditions in a reservoir in northeastern Brazil. This work characterized the zooplankton and the environmental variables of the Canoas reservoir (Assaré, Ceará, Brazil) through eight consecutive monthly collections during a period of intense drought. Zooplankton samples were collected by vertical plots of $50 \mu \mathrm{m}$ plankton net at three longitudinally distributed points. Five species were found: Brachionus calicyflorus Pallas, 1938 (Brachionidae: Rotifera), Diaphanosoma spinulosum Herbst, 1967 (Sididae: Cladocera), Thermocyclops decipiens Kiefer, 1929 and T. inversus Kiefer, 1936 (Cyclopoida: Copepoda), and Notodiaptomus cearensis Wright, 1936 (Calanoida: Copepoda). Thermocyclops decipiens had the highest relative abundance, followed by $N$. cearensis and B. calicyflorus. The spatial and temporal variation of the zooplankton community can be explained by most of the environmental variables associated with $N$. cearensis and $B$. calicyflorus, while T. decipiens was not associated with any of them. The low species richness and dominance of T. decipiens and B. calicyflorus may be a reflection of the high trophic condition of the reservoir during severe drought, and $N$. cearensis is also associated with these more adverse conditions.
\end{abstract}

KEYWORDS. Bioindicator, distribution, community structure, zooplankton, semiarid.

RESUMO. Esse trabalho caracterizou o zooplâncton e as variáveis ambientais do reservatório Canoas (Assaré, Ceará, Brasil) através de oito coletas consecutivas mensais, durante um período de estiagem intensa. Amostras de zooplâncton foram coletadas com arrastos verticais de rede de plâncton de $50 \mu \mathrm{m}$ em três pontos distribuídos longitudinalmente. Foram encontradas cinco espécies: Brachionus calicyflorus Pallas, 1938 (Brachionidae: Rotifera), Diaphanosoma spinulosum Herbst, 1967 (Sididae: Cladocera), Thermocyclops decipiens Kiefer, 1929 e T. inversus Kiefer, 1936 (Cyclopoida: Copepoda), e Notodiaptomus cearensis Wright, 1936 (Calanoida: Copepoda). Thermocyclops decipiens apresentou a maior abundância relativa, seguido de N. cearensis e $B$. calicyflorus. A variação espacial e temporal da comunidade zooplanctônica pode ser explicada pela maioria das variáveis ambientais associadas a $N$. cearensis e $B$. calicyflorus, enquanto $T$. decipiens não se associou a nenhuma delas. A baixa riqueza de espécies e a dominância de $T$. decipiens e $B$. calicyflorus podem ser reflexos da elevada condição trófica do reservatório, em período de estiagem intensa, e $N$. cearensis está associado também com essas condições mais adversas.

PALAVRAS-CHAVE. Bioindicador, distribuição, estrutura da comunidade, zooplâncton, semiárido.

A região semiárida é caracterizada por condições climáticas e hidrológicas peculiares, apresentando longos períodos de estiagem, poucas e curtas chuvas, elevada evaporação e consequentemente, tempo de residência hídrico longo (ChelLAPPA et al., 2009). As características climáticas da região, que se manifestam em ciclos anuais de seca e chuva, têm como consequência grandes oscilações no volume de água acumulado nos reservatórios de abastecimento, provocando modificações da estabilidade da coluna d'água e mudanças na composição das comunidades de organismos aquáticos (DANTAS et al., 2012).
Entre as flutuações que ocorrem nos ecossistemas aquáticos há a perda ou o aumento de biodiversidade, especialmente em situações extremas como sob a interferência humana ou eventos naturais, por exemplo, estiagens e inundações (TUNDISI, 2003). Através da diminuição do nível da água que ocorrem nos reservatórios, eventos de seca afetam as características limnológicas, promovendo variações no oxigênio dissolvido e $\mathrm{pH}$, aumento da condutividade e alcalinidade, aumento da concentração de nutrientes, diminuição da zona eufótica e aumento da biomassa algal (Brasil et al., 2016; FigueIREdo \& BECKER, 2018). A inter- 
relação entre esse conjunto de fatores são refletidas pelo zooplâncton, podendo ser observadas em seus atributos ecológicos (LORETO \& OKAMO, 2007).

Muitas espécies zooplanctônicas são bons indicadores da qualidade de água por apresentarem um curto ciclo de vida e boa sensibilidade às variáveis comumente mensuradas, refletindo de forma rápida às mudanças provocadas por ações antrópicas, como o enriquecimento de nutrientes (Matsumura-Tundisi \& TUNDISI, 2003; LANDA et al., 2007). Diversos estudos apontam as espécies de copépodes do gênero Thermocyclops como bastante sensíveis às perturbações tróficas (LANDA et al., 2007; Perbiche-Neves et al., 2016), e também algumas espécies de rotíferos e cladóceros (GHIDINI et al., 2009; SERAFIM-JÚNIOR et al., 2010).

$O$ fenômeno da eutrofização tende a se intensificar com as mudanças climáticas devido ao consequente aumento da temperatura associado à redução da precipitação nos meses secos (Paerl \& Huisman, 2009; Moss et al., 2011; Brasil et al., 2016). As implicações remetem em um clima mais quente, onde os níveis das concentrações de nutrientes precisam ser reduzidos substancialmente em muitos lagos para que a eutrofização artificial seja controlada (Kosten et al., 2012). O aumento da biomassa algal, principalmente florações de cianobactérias, é o indício mais comum da eutrofização, as demais consequências, estão relacionadas diretamente à proliferação das algas (REGO et al., 2019).

Estas características são comumente observadas em reservatórios da região Nordeste do Brasil, e em resposta a isso, em seis reservatórios no semiárido a maioria das espécies de zooplâncton encontradas são de ambientes eutrofizados (ESKINAZI-SANT'ANNA et al., 2007; Sousa et al., 2008; AlmEIDA et al., 2009). Entretanto, a maioria dos trabalhos já feitos em reservatórios na região Nordeste do Brasil sobre zooplâncton teve como enfoque a composição taxonômica e diversidade específica (ALMEIDA et al., 2010). Para o Estado do Ceará, há a contribuição de LeITÃo et al. (2003), conduzido em três reservatórios urbanos (Pacajus, Pacoti e Gavião).

Assim, o estudo visa identificar a composição de espécies do zooplâncton e sua relação com os fatores ambientais estruturantes da comunidade em um reservatório tropical do semiárido durante um período de intensa estiagem, com o volume bastante reduzido. Devido ao elevado grau trófico espera-se diminuição na riqueza de espécies e simplificação da comunidade, com variações irregulares da riqueza (MATSUMURA-TUNDISI \& TUNDISI, 2005; ZAGANINI et al., 2011). A comunidade zooplanctônica será também analisada quanto ao seu potencial uso como indicadora de condições de elevada trofia, com base em outros estudos que apontam potenciais espécies para esse uso. Adicionalmente, é o primeiro estudo sobre o zooplâncton realizado nesse reservatório.

\section{MATERIAL E MÉTODOS}

O reservatório localiza-se na Bacia Hidrográfica do Alto Rio Jaguaribe no município de Assaré, Ceará, Brasil
( $\left.06^{\circ} 56^{\prime} 48,08^{\prime \prime} \mathrm{S}, 39^{\circ} 56^{\prime} 27,29^{\prime \prime} \mathrm{W}\right)$ e abastece toda a sua população, com aproximadamente 23.191 habitantes (IPECE, 2017). A região apresenta clima tropical quente (semiárido), com pluviosidade média de aproximadamente $680,7 \mathrm{~mm}$ por ano. A temperatura média varia entre 24 e $26^{\circ} \mathrm{C}$, e o período chuvoso ocorre em fevereiro, março e abril. $\mathrm{O}$ tipo de relevo é maciço residual, depressão sertaneja, com solos litólicos, latossolo vermelho-amarelo, podzólico vermelho-amarelo e terra roxa estruturada similar (SANTANA, 2009; IPECE, 2017).

$\mathrm{O}$ reservatório Canoas possui área de $40 \mathrm{~km}^{2}$, profundidade máxima de $50 \mathrm{~m}$ na barragem e volume de $69.250 .000 \mathrm{~m}^{3}$. Durante o estudo toda a região passou por um período de estiagem intensa, sendo observada apenas diminuição do nível da água no reservatório durante as coletas (FUNCEME, 2017; COGERH, 2017a).

Foram escolhidos três pontos de amostragem no reservatório: $\mathrm{P} 1$, localizado próximo à Barragem (06 $56^{\circ} 46$. 9” S, 39॰56'26.1”W); P2 (06 57'15.8”'S, 39 56'25.1”W) e P3 (06 $57^{\prime} 06.5^{\prime}$ 'S, $039^{\circ} 55^{\prime} 59.4^{\prime}$ N), ambos na região intermediária, num eixo longitudinal (Fig. 1).

As coletas foram realizadas mensalmente entre outubro de 2016 e maio de 2017, totalizando oito meses. Esse intervalo abrangeu os períodos considerados como seco e chuvoso, porém a pluviosidade foi abaixo do esperado (COGERH, 2017b; FUNCEME, 2017). O zooplâncton foi coletado com uma rede de plâncton com $50 \mu \mathrm{m}$ de abertura de malha e boca de $30 \mathrm{~cm}$. Foram feitos arrastos verticais $(\mathrm{n}=24)$ para amostras quantitativas, e horizontais e verticais $(\mathrm{n}=24)$ para as qualitativas. Foi obtido um total de 48 amostras (quantitativas e qualitativas).

O material alocado foi fixado em solução de formol a $10 \%$ e as amostras depositadas no Laboratório de Crustáceos do Semiárido (LACRUSE), da Universidade Regional do Cariri - URCA. A identificação das espécies se deu através de microscopia óptica, com a utilização de estiletes com ponta fina para dissecar espécimes de Copepoda. Foram usadas as seguintes referências para a identificação dos táxons: RocHA \& Tundisi (1976), Koste (1978), ReId (1985), Dumont (1995); Elmoor-Loureiro (1997), Ueda \& ReId (2003), Perbiche-Neves et al. (2015).

A densidade numérica dos indivíduos (copépodes, cladóceros e rotíferos) foi estimada a partir da contagem dos mesmos, em duas subamostragens em uma câmera de contagem (Sedgewick-Rafter de $1 \mathrm{~mL}$ para rotíferos e náuplios e rotíferos; e cubeta de acrílico para cladóceros e copépodes adultos), até atingir um total de no mínimo 200 indivíduos ao todo por amostra. A densidade final foi expressa em ind. $/ \mathrm{m}^{-3}$. $\mathrm{O}$ valor foi obtido pela fórmula do volume do cilindro.

Para os dados abióticos foram utilizadas sondas específicas: Temperatura da água $\left({ }^{\circ} \mathrm{C}\right.$, HANNA HI 9146);

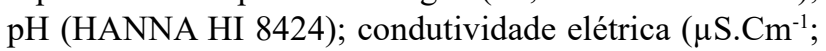
HANNA HI 99300); sólidos totais dissolvidos (Mg.L $\mathrm{L}^{-1}$; HANNA HI 99300); oxigênio dissolvido (Mg.L ${ }^{-1}$; HANNA HI 9146); e a transparência da água (cm; disco de Secchi).

Os dados de abundância do zooplâncton e das variáveis físicas e químicas foram correlacionados entre si 


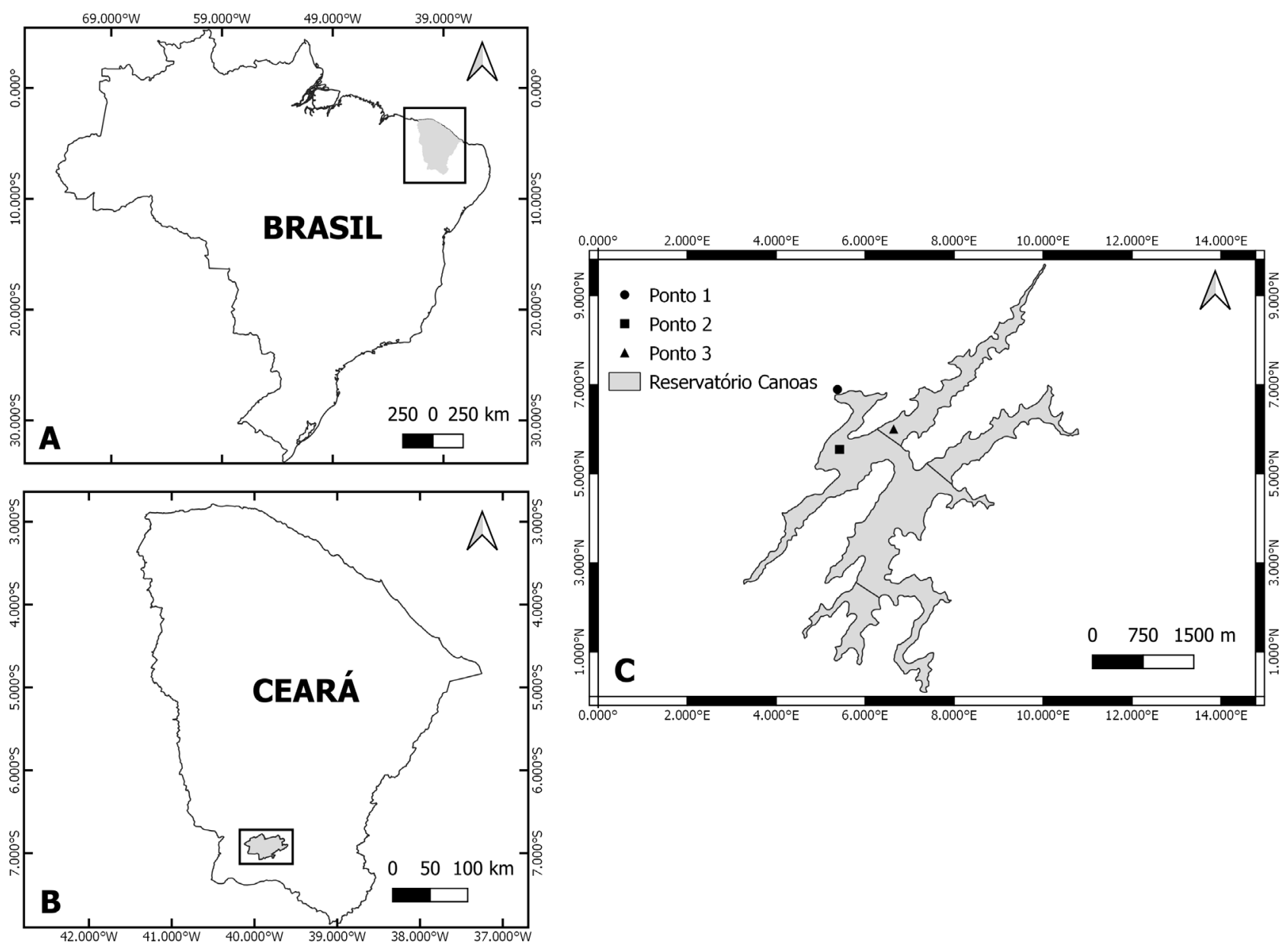

Fig. 1. Localização da área de estudo: A, Brasil; B, Estado do Ceará com destaque para o município de Assaré; C, Reservatório Canoas e os respectivos pontos onde foram realizadas as coletas.

usando uma análise de ordenação, a de redundância (RDA). Os dados foram transformados em $\log \mathrm{X}+1$, com exceção do $\mathrm{pH}$. Essa análise foi feita no software R Cran Project 3.3.4 (2018), usando o pacote vegan (OKSANEN et al., 2019). As correlações significativas $(\mathrm{P}<0,05)$ foram consideradas no biplot.

\section{RESULTADOS}

Durante o estudo foi destaque o processo de redução de volume do reservatório, especialmente por elevada evaporação e baixa precipitação. Entre as variáveis limnológicas, destacam-se os elevados valores de condutividade elétrica (média: $717 \pm 30 \mu \mathrm{S} . \mathrm{cm}^{-1}$ ). $\mathrm{O} \mathrm{pH}$ foi básico (média: $8,6 \pm 0,71$ ), e a média da temperatura da água no período foi alta $\left(29,3 \pm 1,41^{\circ} \mathrm{C}\right)$, bem como a concentração média de oxigênio dissolvido nas camadas superficiais $(8,7 \pm 1,2 \mathrm{mg}$.L-1). A transparência da água foi baixa durante todo o período de estudo $(26,7 \pm 1,94 \mathrm{~cm})$. Os sólidos totais dissolvidos apresentaram baixa variação no período estudado (359 $\pm 14,4$ mg.L-1 $)$.
A pluviosidade acumulada anual média para a região no período estudado é de $700 \mathrm{~mm}$, e no ano em que as amostragens foram feitas o total acumulado foi aproximadamente $415 \mathrm{~mm}$ (Fig. 2). No início do estudo o volume de água armazenado no Canoas correspondia a cerca de $9,57 \%$ da capacidade máxima do reservatório, atingindo um volume equivalente a $8,67 \%$ da sua capacidade total no final do período.

Em relação ao zooplâncton foram encontradas cinco espécies, com valor médio de $3 \pm 1$ espécies por amostra. Observou-se uma tendência de diminuição no número de espécies e da abundância total no decorrer do estudo (Fig. 3), conforme houve aumento da intensidade da estiagem.

Copepoditos de Cyclopoida foram dominantes sobre as demais formas imaturas de Copepoda. Náuplios dessa mesma ordem também foram expressivos (Fig. 4). Entre as espécies destaca-se a elevada abundância dos copépodes Thermocyclops decipiens e Notodiaptomus cearensis, seguido do rotífero Brachionus calicyflorus (Fig. 5). A partir de janeiro T. decipiens registrou aumento expressivo de abundância, persistindo como dominante até o mês de abril. Entre outubro 


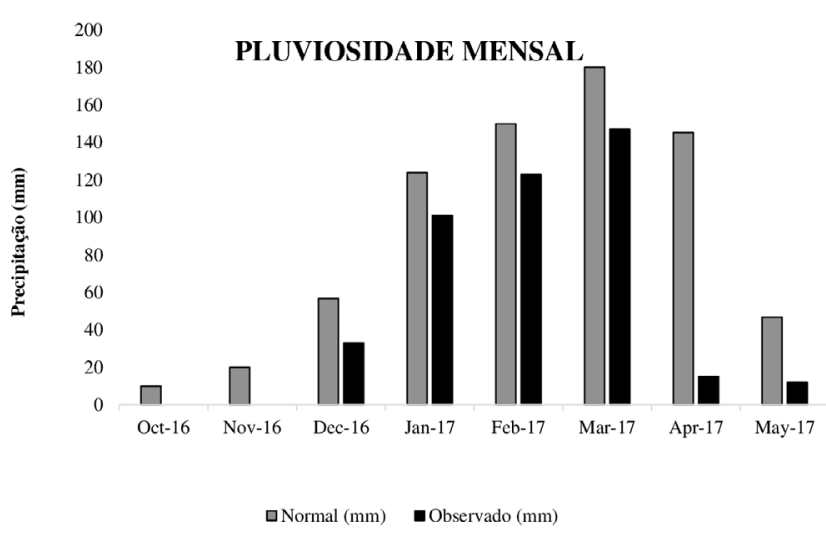

Fig. 2. Precipitação acumulada entre os meses de outubro a dezembro de 2016 e janeiro a maio de 2017 no reservatório Canoas, Ceará, Brasil (Portal Hidrológico do Ceará), junto com os valores esperados normalmente.

e dezembro B. calicyflorus e $N$. cearensis apresentaram elevadas abundâncias, similares ou maiores a $T$. decipiens.

A análise de redundância canônica explicou ao todo $52 \%$ da correlação entre a abundância dos táxons e as variáveis ambientais (Fig. 6). As variáveis temperatura da água, oxigênio dissolvido, condutividade elétrica, sólidos totais em suspensão, transparência e $\mathrm{pH}$ foram positivamente correlacionadas com B. calicyflorus e $N$. cearensis e meses do auge do período seco (outubro a dezembro). As outras três espécies (D. spinulosum, T. inversus e T. decipiens) foram correlacionadas inversamente a essas variáveis, junto aos meses do período chuvoso (fevereiro a maio).

\section{DISCUSSĀO}

Os resultados indicaram $B$. calicyflorus e $N$. cearensis como dominantes no ambiente eutrofizado e em processo de estiagem. As elevadas abundâncias dessas espécies foram correlacionadas com o aumento da temperatura da água, oxigênio dissolvido e condutividade. Durante as florações de algas intensas pode haver o acúmulo de oxigênio dissolvido nas camadas superficiais, porém ocorre anoxia nas camadas mais profundas (VIEIRA et al., 2015). A temperatura aumenta em períodos de estiagem, com baixa pluviosidade pela redução do volume por evaporação e acúmulo de calor no lago (EsTEVEs, 2011). Isso pode potencializar os efeitos de concentração de nutrientes, íons, estabilidade duradoura na coluna de água e a proliferação de algas e cianobactérias, selecionando consequentemente as espécies zooplanctônicas presentes e tornando a comunidade simplificada e resistente às condições mais adversas (MATSUMURA \& TUNDISI, 2005; ZAGANINI et al., 2011; REGo et al., 2019).

Embora T. decipiens não tenha sido correlacionado com nenhuma variável, sua dominância e presença durante todo o estudo também apontam como eficiente indicador de condições eutróficas, estando adaptado e prevalecendo nessas condições conforme observado em outros estudos no Brasil (Silva \& Matsumura-Tundisi, 2005; LANDA et al., 2007; Perbiche-Neves et al., 2016). A ausência de correlações com qualquer variável pode ser explicada pela sua frequência de

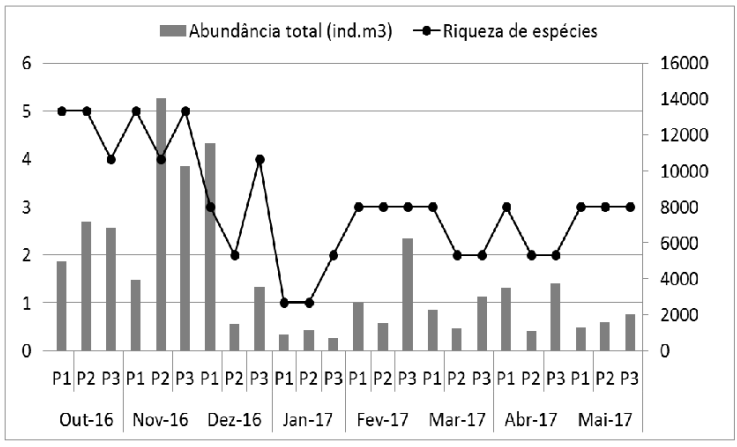

3

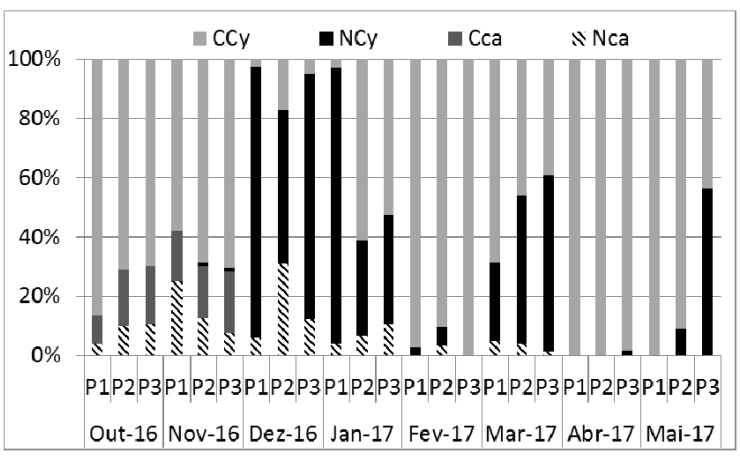

4

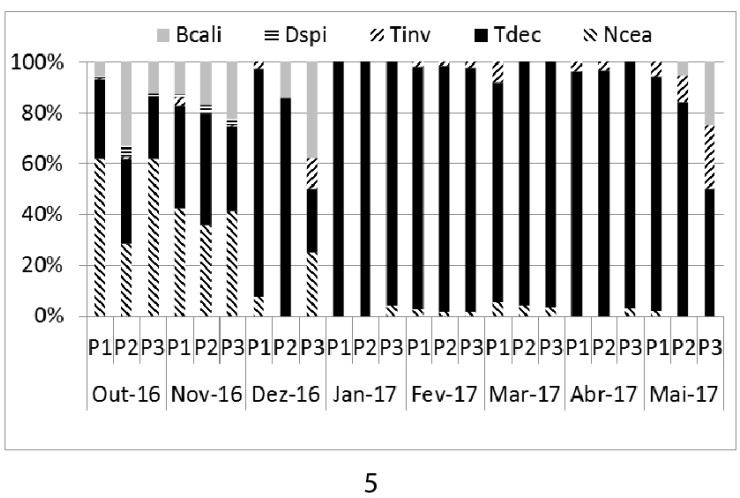

Figs 3-5: Fig. 3, variação espacial e temporal da riqueza de espécies e da abundância total; Fig. 4, variação da abundância relativa de formas jovens de Copepoda (CCy, Copepoditos de Cyclopoida; NCy, Náuplios de Cyclopoida; Cca, Copepoditos de Calanoida; Nca, Náuplios de Calanoida); Fig. 5, variação da abundância relativa das espécies encontradas, formas adultas (Bcali, Brachiounus calicyflorus; Dspi, Diaphanosoma spinulosum; Tinv, Thermocyclops inversus; Tdec, Thermocyclops decipiens; Ncea, Notodiaptomus cearensis).

ocorrência alta entre as amostras coletadas, estando presente quase que durante todo o período estudado.

Assim, essas três espécies acima citadas $(B$. calicyflorus, $T$. decipiens e $N$. cearensis) indicam condições eutróficas e de estiagem, corroborando com informações do sistema de monitoramento do reservatório e com outros estudos que encontraram resultados similares (LANDA et al., 2007; Perbiche-Neves et al., 2007, 2016; SERAFIM-JúNIOR et al., 2010; Silva, 2011).

Notodiaptomus cearensis tolera ampla faixa de condutividade, suportando concentrações elevadas de sais 


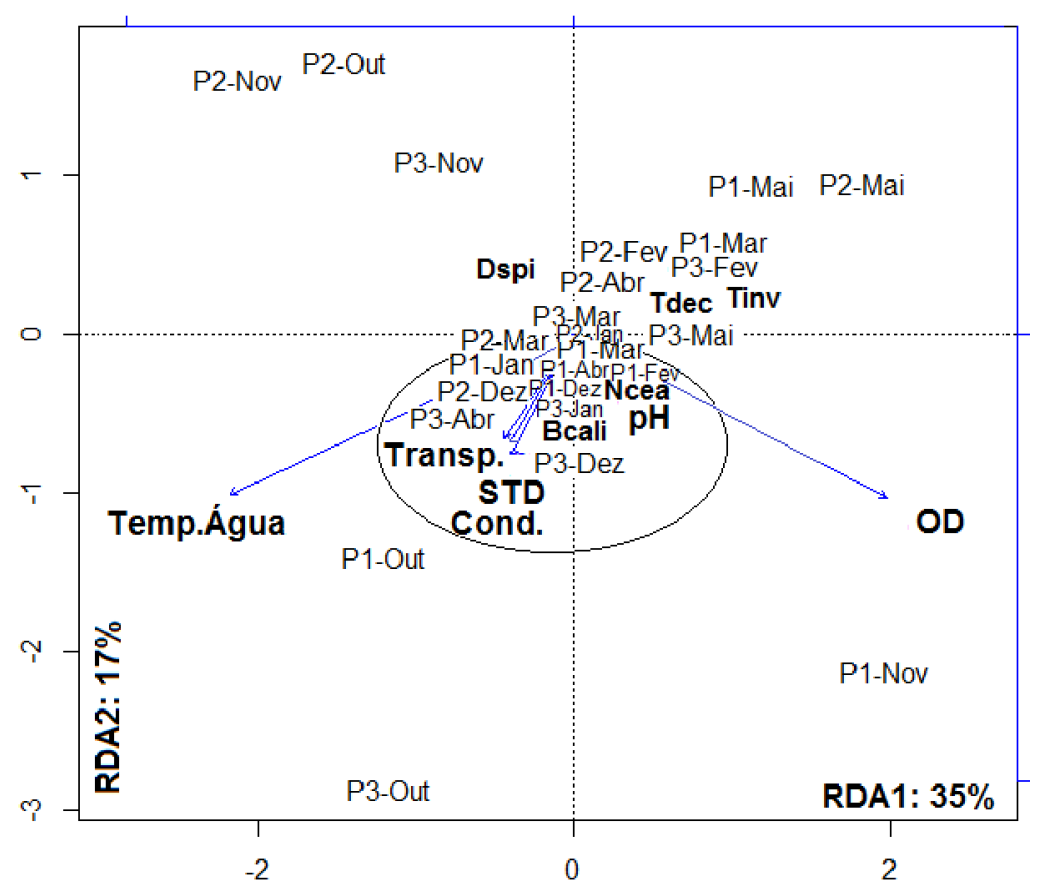

Fig. 6. RDA entre os dados bióticos e abióticos nos respectivos pontos (P1, P2 e P3) e meses (OD, oxigênio dissolvido; pH, potencial hidrogeniônico; Transp, transparência da água; Temp.Água, temperatura da água; Cond., condutividade elétrica; STD, sólidos totais dissolvidos. Espécies: Ncea, $N$. cearensis; Tdec. T. decipiens; Tinv, T. inversus; Dspi, D. spinulosum; Bcali, B. calyciflorus).

como a do presente estudo, na faixa de $700 \mu \mathrm{S} . \mathrm{cm}^{-1}$, cuja concentração pode ser limitante para outras espécies de copépodes calanóides. Matsumura-Tundisi \& TUNDisI (2003) apontam para trocas de espécies de copépodes diaptomídeos associadas aos aumentos de condutividade elétrica. No reservatório de Barra Bonita, Rio Tietê (SP, Brasil), a condutividade variou entre 150 e $270 \mu{\mathrm{S} . \mathrm{cm}^{-1} \mathrm{em}}$ medições realizadas em 1985 e 1986 (MATSUMURA-TUNDISI \& TUNDISI, 2005), com redução no ano de 1995 (SANTosWISNIEWSKI \& ROCHA, 2007), entre 100 e $160 \mu{\mathrm{S} . \mathrm{cm}^{-1}}^{-1}$ aproximadamente, e aumento para valores médios de 550 $\mu \mathrm{S} . \mathrm{cm}^{-1}$ em medições realizadas em 2007 e 2008, em um ponto mais a montante no reservatório, na desembocadura do rio Tietê (ZAGANINI et al., 2011).

Nos três estudos acima citados houve dominância de B. calicyflorus e T. decipiens, entre outras, porém em 2010 $N$. cearensis foi detectada no corpo central do reservatório (Perbiche-Neves et al., 2014). No presente estudo parece ser a única espécie de Calanoida ocorrente no período em que o reservatório foi estudado, suportando os trabalhos que associam essa espécie com ambientes eutrofizados ou com condições mais severas, como as de estiagem e temperaturas elevadas.

A dominância de T. decipiens mais uma vez indica a sua eficiência em relacionar-se com águas eutróficas, já apontadas por outros estudos (LANDA et al., 2007; Silva, 2011). Ainda, a maior proporção de náuplios e copepoditos de Cyclopoida em relação à Calanoida reforçam outros trabalhos que apontam essa tendência como indicadora de estado trófico em reservatórios (SENDACZ et al., 2006).
Thermocyclops inversus tende a ocorrer em ambientes mesotróficos, podendo ocorrer em ambientes eutróficos (SILVA, 2011; Perbiche-Neves et al., 2016). Landa et al. (2007) também encontraram resultados similares em reservatórios do estado de Minas Gerais. A baixa abundância verificada nesse estudo pode ser explicada pelo estado eutrófico o qual encontra-se o reservatório Canoas (COGERH, 2018).

Brachionus calicyflorus é dominante em reservatórios eutróficos (Perbiche-Neves et al., 2012), sendo um indicador desses tipos de ambientes por sua frequência e abundância. Sua presença é associada com florações de algas cianobactérias (SERAFIM-JÚNIOR et al., 2010). Por outro lado, Diaphanosoma spinulosum tende a ser comum em reservatórios, independente do estado trófico (PERBICHENeves \& NogueIra, 2010), sendo organismos adaptados à vida planctônica.

Embora neste estudo a estrutura da comunidade zooplanctônica seja simples em número de espécies, as espécies registradas responderam ao estado hipereutrófico do reservatório, em processo de redução de volume devido a intensa estiagem. As espécies dominantes são típicas de ambientes eutróficos conforme a literatura disponível. Condições de estiagem que ocasionem estresse ambiental tendem a funcionar como filtros na seleção das espécies dominantes.

Agradecimentos. Os autores agradecem a Universidade Regional do Cariri (URCA) e as equipes do Laboratório de Crustáceos do Semiárido (LACRUSE) e do Laboratório de Botânica (LaB) desta mesma IES, pela disponibilidade de espaço e materiais necessários a realização desta pesquisa. 


\section{REFERÊNCIAS}

Almeida, V. L. S.; Dantas, E. W.; Melo-Júnior, M.; BittencourtOliveira, M. C. \& Moura, A. N. 2009. Zooplanktonic Community of Six Reservoirs in Northeast Brasil. Brazilian Journal of Biology 69(1):57-65.

Almeida, V. L. S.; Júnior, M. M.; Paranaguá, M. N.; Larrazábal, M. E. L \& Melão, M. G. G. 2010. O Zooplâncton de Água Doce e Seu Estudo em Reservatórios do Nordeste do Brasil. Reservatórios do Nordeste do Brasil: Biodiversidade, Ecologia e Manejo. Canal 6(1):441-475.

Brasil, J.; Attayde, J. L.; Vasconcelos, F. R.; Dantas, D. D. F. \& Huszar, V. L. M. 2016. Drought-induced water-level reduction favors cyanobacteria blooms in tropical shallow lakes. Hydrobiologia770(1):145-164.

Chellappa, N. T.; Chellappa, T.; CÂmara, F. R.; Rocha, O. \& Chellappa, S. 2009. Impact of stress and disturbance factors on the phytoplankton communities in Northeastern Brazil reservoir. Limnologica 39(4):273-282.

COGERH. 2017a. Portal Hidrológico do Ceará: Volume dos Reservatórios (Reservatório Canoas). Disponível em $<$ http://www.hidro.ce.gov.br/ reservatorios/volume>. Acessado em junho 10, 2017.

COGERH. 2017b. Sub-bacia Hidrográfica do Alto Jaguaribe Características Gerais. Disponível em $<$ https:/www.srh.ce.gov.br/ wp-content/uploads/sites/90/2018/07/Caracteriza $\%$ C3\%A7\%C3\%A3oda-Bacia-Hidrogr\%C3\%A1fica-Alto-Jaguaribe.pdf>. Acessado em junho 10, 2017.

COGERH. 2018. Portal Hidrológico do Ceará: Monitoramento da Eutrofização (Reservatório Canoas). Disponível em < http://www. hidro.ce.gov.br/reservatorios/qualidade>. Acessado em janeiro 10, 2018.

Dantas, E. W.; Bittencourt-Oliveira, M. do C. \& Moura, A. do N. 2012. Dynamics of phytoplankton associations in three reservoirs in northeastern Brazil assessed using Reynolds' theory. Limnologica 42(1):72-80.

Dumont, J. H. 1995. Guides to of Identification of The Micro Invertebrates of The Continental Waters of The World. Ghent, State University of Ghent.

ElmoOR-Loureiro, L. M. A. 1997. Manual de Identificação de Cladóceros Límnicos do Brasil. Brasília, Universa. 156p.

Eskinazi-Sant'anna, E. M. E. S.; Menezes, R.; Costa, I. A. S.; Panosso, R. F.; Araúuo, M. F. \& Attayde, J. L. 2007. Composição da Comunidade Zooplanctônica em Reservatórios Eutróficos do Semiárido do Rio Grande do Norte. Oecologia Brasiliensis 11(3):410-421.

Esteves, F. A. 2011. Fundamentos de Limnologia. 3ed. Rio de Janeiro, Interciência, 826p.

FigueIREDo, A. V. \& BECKER, V. 2018. Influence of extreme hydrological events in the quality of water reservoirs in the semi-arid tropical region. RBRH23:e53.

FUNCEME. 2017. Pluviosidade da Região Hidrográfica do Alto Jaguaribe. Disponível em <http://www.funceme.br/index.php/tempo/ chuvasmensais-regiãohidrográfica>. Acessado em junho 10, 2017.

Ghidini, A. R.; Serafim-Júnior, M.; Perbiche-Neves, G. \& Brito, L. 2009. Distribution of Planktonic Cladocerans (Crustacea: Branchiopoda) of a Shallow Eutrophic Reservoir (Paraná State, Brazil). Pan-American Journal of Aquatic Sciences 4:294-305.

IPECE. 2017. Instituto de Pesquisa e Estratégia Econômica do Ceará. Perfil Básico Municipal 2016 Assaré. Disponível em < http://www. ipece.com.br>. Acessado em junho 10, 2017.

Koste, W. 1978. Rotatoria Die Rodertiere Itteleuropas Begtündet Von Max Voigt - Monogononta. 2. Auflage Neubearbeitet Von Walter Koste. Berlin, Gebrüder Borntraeger, vol. 1-2.

Kosten, S.; Huszar, V. L. M.; Bécares, E.; Costa, L. S.; Van Donk, E.; Hansson, L. A.; Jeppesen, E.; KruK, C.; Lacerot, G.; Mazzeo, N.; De Meester, L.; Moss, B.; Lürling, M.; Nõges, T.; Romo, S. \& SCHEFFER, M. 2012. Warmer climates boost cyanobacterial dominance in shallow lakes. Global Change Biology 18(1):118-126.

Landa, G. G.; Barbosa, F. A. R.; Rietzler, A. C. \& Maia Barbosa, P. M. 2007. Thermocyclops decipiens (Kiefer, 1929) (Copepoda, Cyclopoida) as Indicator of Water Quality in the State of Minas Gerais, Brazil. Brazilian Archives of Biology and Technology 50:695-705.

Leitão, A. C.; Freire, R. H. F. \& Rocha, O. 2003. Composição e Variação Sazonal da Comunidade Zooplanctônica em Três Reservatórios da Região Metropolitana de Fortaleza, Ceará, Brasil. In: Anais do VI Congresso de Ecologia do Brasil, Fortaleza, Sociedade de Ecologia do Brasil, p. 164-166.
Loreto, R. G. \& OKano, W. Y. 2007. Dinâmica Estrutural da Comunidade Zooplanctônica na Criação de Tilápia do Nilo em Diferentes Manejos Alimentares. In: Anais do VIII Congresso de Ecologia do Brasil, Caxambu, Sociedade de Ecologia do Brasil, p. 1-2.

Matsumura-Tundisi, T. \& Tundisi, J. G. 2003. Calanoida (Copepoda) Species Composition Changes in the Reservoirs of São Paulo State (Brazil) in the Last Twenty Years. Hydrobiologia 504:215- 222.

Matsumura-Tundisi, T. \& Tundisi, J. G. 2005. Plankton Richness in a Eutrophic Reservoir (Barra Bonita Reservoir, SP, Brazil). Hydrobiologia 542:367-378.

Moss, B.; Kosten, S.; Meerhoff, M.; Battarbee, R. W.; Jeppesen, E.; Mazzeo, N.; Havens, K.; Lacerot, G.; LiU, Z.; De Meester, L.; Paerl, H. \& SCHEFFER, M. 2011. Alliedattack: climatechangeandeutrophication. Inland Waters 1:101-105.

Oksanen, J.; Blanchet, F. G.; Friendly, M.; Kindt, R.; Legendre, P.; McGlinn, D.; Minchin, P. R.; O’hara, R. B.; Simpson, G. L.; Solymos, P.; Stevens, M. H. H.; Szoecs, E. \& Wagner, H. 2019. Package 'vegan'. R Cran Project. Disponível em $<$ https://cran.r-project.org, https://github.com/vegandevs/vegan>. Acessado em janeiro 05, 2019.

Paerl, H. W \& Huisman, J. 2009. Climatechange: a catalyst for global expansion of harmful cyanobacterial blooms. Environmental MicrobiologyReports1(1):27-37.

Perbiche-Neves, G. \& Nogueira, M. G. 2010. Multidimensional Effects on Cladoceran (Crustacea, Anomopoda) Assemblages in Two Cascade Reservoirs (SE - Brazil). Lakes and Reservoirs: Research and Management 15(2):151-164.

Perbiche-Neves, G.; Boxshall, G. A.; Previattelli, D.; Nogueira, M. G. \& DA Rocha, C. E. F. 2015. Identification Guide to Some Diaptomid Species (Crustacea, Copepoda, Calanoida, Diaptomidae) of "de la Plata” River Basin (South America). ZooKeys 497:1-111.

Perbiche-Neves, G.; Nogueira, M. G.; Oliveira, P. \& Serafim-Júnior, M. 2014. Copépodes (Crustacea, Calanoida, Cyclopoida) Planctônicos em Reservatórios Profundos e Dendríticos: efeitos do tempo de residência e da idade de formação. Brazilian Journal of Aquatic Science and Technology 18(1):1-8.

Perbiche-Neves, G.; Serafim-Júnior, M.; Ghidini, A. R. \& Brito, L. 2007. Spatial and Temporal Distribution of Copepoda (Cyclopoida and Calanoida) of an Eutrophic Reservoir in The Basin of Upper Iguaçu River, Paraná, Brazil. Acta Limnologica Brasiliensia 19(4):393-406. Perbiche-Neves, G.; Serafim-Júnior, M.; Portinho, J. L.; Shimabukuro, E. M.; GHIDINI, A. R. \& BRITo, L. 2012. Effect of Atypical Rainfall on Lotic Zooplankton: comparing downstream of a reservoir and tributaries with free stretches. Tropical Ecology 53(2):149-162.

Perbiche-Neves, G.; Saito, V. S.; Previattelli, D.; Da Rocha, C. E. \& Nogueira, M. G. 2016. Cyclopoid Copepods as Bioindicators of Eutrophication in Reservoirs: Do patterns hold for large spatial extents? Ecological Indicators (70):340-347.

Rego, A. H. G.; Rangel-Junior, A. \& Costa, I. A. S. 2019. Phytoplankton scenario and microcystin in water during extreme drought in semiarid tropical water supplies, Northeastern Brazil. Brazilian Journal of Biology 80(1):1-11.

REID, J. W. 1985. Chave de Identificação Para as Espécies Continentais Sul Americanas de Vida Livre da Ordem Cyclopoida (Crustacea, Copepoda). Boletim de Zoologia, Universidade de São Paulo 9:17-143.

Rocha, O. \& Tundisi, T. M. 1976. Atlas do Zooplâncton: Copepoda. São Paulo, Universidade Federal de São Carlos. 68p.

Santana, E. W. coord. 2009. Caderno regional da sub-bacia do Alto Jaguaribe: Conselho de Altos Estudos e Assuntos Estratégicos. Fortaleza: INESP (Coleção Cadernos Regionais do Pacto das Águas), 5(1):1-131.

Santos-Wisniewski, M. J. \& Rocha, O. 2007. Spatial Distribution and Secundary Production of Copepoda in a Tropical Reservoir: Barra Bonita, SP, Brazil. Brazilian Journal of Biology 67(2):223-233.

Sendacz, S.; CalefFi, S. \& Santos-Soares, J. 2006. Zooplankton Biomass of Reservoirs in Different Trophic Conditions in The State of São Paulo, Brazil. Brazilian Journal of Biology 66(1B):337-350.

Serafim-Júnior, M.; Perbiche-Neves, G.; Brito, L. D.; Ghidini, A. R. \& CASAnova, S. 2010. Variação Espaço-Temporal de Rotifera em um Reservatório Eutrofizado no Sul do Brasil. Iheringia, Série Zoologia 100(3):233-241.

Silva, W. M \& Matsumura-Tundisi, T. 2005. Taxonomy, Ecology, and Geographical Distribution of The Species of the Genus Thermocyclops 
Kiefer, 1927 (Copepoda, Cyclopoida) in Sao Paulo State, Brazil, with description of a new species. Brazilian Journal of Biology 65(3):521-531.

SiLva, W. M. 2011. Potential Use of Cyclopoida (Crustacea, Copepoda) as Trophic State Indicators in Tropical Reservoirs. Oecologia Australis 15(3):511-521.

Sousa, W.; Attayde, J. L.; Rocha, E. S. \& Eskinazi-Sant'anna, E. M. 2008. The Response of Zooplnkton Assemblages to Variations in the Water Quality of Four Man-Made Lakes in Semiarid Northeastern Brazil. Journal of Plankton Research 30(6):699-708.

Tundisi, J. G. 2003. Água no Século XXI: enfrentando a escassez. In: TundisI, J. G. org. Água no Século XXI: enfrentando a escassez. São Carlos, RiMa, p. 1-15.
UedA, H. \& ReID, J. W. 2003. Copepoda: Cyclopoida - Genera Mesocyclops and Thermocyclops. In: DumONT, H. J. F. ed. Guides to the Identification of the Microinvertebrates of the Continental Waters of the World. The Netherlands, Backhuys Publishers, p. 1-316.

Vieira, P. C. S.; Cardoso, M. M. L. \& Costa, I. A. S. 2015. Vertical and temporal dynamics of phytoplanktonic associations and the application of index assembly in tropical semi-arid eutrophic reservoir, northeastern Brazil. Acta Limnologica Brasiliensia 27(1):130-144.

Zaganini, R. L.; Perbiche-Neves, G. \& Naliato, G. D. A. O. \& Carvalho, E. D. 2011. Baixa Diversidade de Zooplâncton na Desembocadura de uma Represa Eutrófica (SP, Brasil): reflexo da poluição? Estudos de Biologia 32/33:76-81. 